\title{
How reliable are left ventricular ejection fraction cut offs assessed by echocardiography for clinical decision making in patients with heart failure?
}

\author{
Beat A. Kaufmann • Son Y. Min • Kaatje Goetschalckx • \\ Alain M. Bernheim • Peter T. Buser • \\ Matthias E. Pfisterer · Hans-Peter Brunner-La Rocca
}

Received: 14 February 2012/Accepted: 29 August 2012/Published online: 11 September 2012

(C) Springer Science+Business Media, B.V. 2012

\begin{abstract}
We aimed to study the potential influence of the variability in the assessment of echocardiographically measured left ventricular ejection fraction (LVEF) on indications for the implantation of internal cardioverter defibrillator and/or cardiac resynchronization devices in heart failure patients. TIME-CHF was a multicenter trial comparing NT-BNP versus symptom-guided therapy in patients aged $\geq 60$ years. Patients had their LVEF assessed at the recruiting centre using visual assessment, the arealength or biplane Simpson's method. Echocardiographic data were transferred to the study core-lab for re-assessment. Re-assessment in the core-lab was done with biplane Simpson's method, and included an appraisal of image quality. 413 patients had the LVEF analyzed at the recruiting centre and at the core lab. Image quality was optimal in 191 and suboptimal in 222. Overall, the correlation between LVEF at the recruiting centres and at the core-lab was good, independent of image quality $\left(\mathrm{R}^{2}=\right.$ $0.62)$. However, when a LVEF $\leq 30 \%$ or $\geq 30 \%$ was used as a cut-off, about $20 \%$ of all patients would have been re-assigned to having either a LVEF above or below the cutoff, this proportion was not significantly influenced by image quality. We conclude that correlation between LVEF assessed by different centres based on the same ultrasound data is good, regardless of image quality. However, one fifth
\end{abstract}

B. A. Kaufmann $(\bowtie) \cdot$ S. Y. Min · K. Goetschalckx ·

A. M. Bernheim - P. T. Buser - M. E. Pfisterer .

H.-P. Brunner-La Rocca

Division of Cardiology, University Hospital Basel, Petersgraben

4, 4031 Basel, Switzerland

e-mail: kaufmannb@uhbs.ch

H.-P. Brunner-La Rocca

Department of Cardiology, Maastricht University Medical

Center, Maastricht, The Netherlands of patients would have been re-assigned to a different category when using the clinically important cut-off of $30 \%$.

Keywords Device therapy - Left ventricular ejection fraction · Variability · Clinical decision-making . Heart failure

\section{Introduction}

Large trials have shown a survival benefit after the implantation of an internal cardioverter defibrillator (ICD) or cardiac resynchronization therapy (CRT) in patients with a severely reduced left ventricular ejection fraction (LVEF) [1-3]. One of the selection criteria in these trials was the reduction of LVEF below a predefined threshold, and transthoracic echocardiography was one of the accepted imaging methods for assessing LVEF. Similarly, guidelines for the treatment of heart failure advocate the initiation of angiotensin converting enzyme inhibitor and $\beta$-blocker therapy using predefined LVEF cut-offs [4, 5]. Therefore, important treatment decisions in this patient group with a high mortality are currently based on the measurement of LVEF by transthoracic echocardiography, both in large randomized clinical trials but also in daily clinical practice.

However, although validated as a prognostic indicator in cardiac diseases $[6,7]$ and successfully used in clinical trials to detect even small changes in ejection fraction in large patient groups [8-10], the measurement of LVEF by 2-dimensional transthoracic echocardiography is fraught with a considerable interobserver variability. 3-dimensional echocardiography and left ventricular opacification with 2 nd generation contrast media have been shown to improve the accuracy of the measurement of LVEF. 
However, despite recommendations by recent guidelines [11], these newer methods are not yet widely used in daily clinical practice. Therefore, assessment of LVEF for the selection of therapies like ICD or CRT is most often performed by 2-dimensional echocardiography. Given the inherent risks of ICD and CRT implantation and the associated costs, the best possible accuracy in measurement of LVEF is warranted. In this study we investigated the variability in the assessment of LVEF with 2-dimensional echocardiography, and the potential impact of this variability on treatment decisions. We also aimed to relate variability in the assessment of LVEF and its effect on treatment decisions to the image quality of the echocardiographic exams and to investigate other potential predictors of variability. We performed this analysis in a large, real world heart failure population.

\section{Methods}

\section{Study population}

TIME-CHF was a multicenter trial comparing an intensified, BNP-guided treatment strategy with a conventional medical treatment strategy in patients aged 60 years or more with heart failure irrespective of LVEF. The design of the TIME-CHF trial has been described elsewhere in detail $[12,13]$. Briefly, patients with dyspnea (New York Heart Association class II or higher on current therapy), a history of hospitalization for heart failure within the past year, and an elevated $\mathrm{N}$-terminal BNP level $(>400 \mathrm{pg} / \mathrm{ml}$ in patients $<75$ years, and $>800 \mathrm{pg} / \mathrm{ml}$ in patients $\geq 75$ years of age) were recruited in 15 tertiary and secondary hospital centers in Switzerland and Germany. Exclusion criteria were dyspnea not mainly due to heart failure, valvular heart disease requiring surgery, acute coronary syndrome within ten days before study inclusion, angina pectoris $>$ CCS 2, revascularization within the month before study inclusion, body mass index $>35$, serum creatinine $>2.49 \mathrm{mg} / \mathrm{dL}$, a life expectancy of less than 3 years due to non-cardiovascular causes, inability to give informed consent, follow-up impossible, or participation in another study. All patients gave written informed consent. The study was approved by the local ethics committee of each participating center. Overall, 622 patients were included in the TIME-CHF trial.

\section{Study protocol}

Upon inclusion in the study, patients had transthoracic echocardiography performed at the recruiting center by a board certified cardiologist trained in echocardiography. Standard clinical ultrasound equipment was used for acquisition of cine loops documenting left ventricular function from parasternal and apical acoustic windows with broadband transducers operating in harmonic imaging mode. The LVEF was determined by the treating cardiologist at the recruiting hospitals either by visual assessment or using tracking of the endothelial border and accepted mathematical models (Biplane Simpson's method or area length method). The echocardiographic studies were stored digitally and transferred to the echocardiography core laboratory at the University Hospital of Basel. Clinical data were derived from the central database of TIME-CHF. Of the 622 patients, $413(66.4 \%)$ had a complete set of echocardiographic images transmitted to the core laboratory, and comprise the patient group for the present study.

At the core laboratory, the LVEF was re-assessed by two readers (SYM and $\mathrm{KG}$ ) blinded to results from the recruiting center. The LVEF was determined from planimetry of cineloops of the apical 4- and 2-chamber windows at end-diastole and end-systole using biplane Simpson's method according to the recommendations of the American Society of Echocardiography [14], leaving the papillary muscles and trabeculations within the cavity. The interobserver variability for these two readers was determined in a randomly selected subset of 30 patients for each of the two reader by re-assessment by a third reader (BAK) blinded to the LVEF values. Interobserver variability was $4.8 \pm 3.8 \%$ for SYM and $4.5 \pm 3.3 \%$ for KG. Regional wall motion was rated for the anterior, inferior, septal lateral, and apical myocardial segments by the same readers on a 5 -point scale $(1=$ normal, $2=$ mild hypokinesia, $2=$ severe hypokinesia, $3=$ akinesia, $4=$ dyskinesia). The presence of a 2 or more points difference in wall motion score between adjacent segments was considered to represent a regional wall motion defect. Image quality was assessed independently by a third investigator (BAK) without knowledge of the LVEF values or clinical details. Image quality was rated as bad when only 50-60\% of the endocardial border could be well visualized in any of the standard apical image planes, as fair when $60-74 \%$ of the endocardial border could be discerned, and as good when $75-100 \%$ of the endocardial border was visible [15]. For the present analysis, subjects were classified into a group with optimal image quality (those classified as having good image quality) and suboptimal image quality (those having bad or fair image quality).

\section{Statistics}

Statistical analyses were performed using SPSS Version 16.0 (SPSS Inc.). Variability was defined as the absolute difference between the two LVEF measurements. Continuous variables were compared between subgroups using a $t$ test or Mann-Whitney test, as appropriate. Categorical variables were compared using Fisher's exact test. 
Correlations were assessed using linear regression analysis, followed by Bland-Altman analysis for assessing the agreement between the two LVEFs. Multivariate linear regression was used for assessment of potential predictors of high interobserver variability. A $p$ value $<0.05$ was considered statistically significant.

\section{Results}

Patient characteristics

The Table 1 shows basic parameters and clinical parameters that may be relevant to echocardiographic image quality. Of the 413 patients included, 191 (46\%) had a good image quality, while 157 (38\%) had a fair image quality and $65(15.7 \%)$ had a bad image quality. Thus, 191 (46\%) of the patients were classified as having an optimal image quality, and $222(54 \%)$ as having a suboptimal image quality. Patients in the group with suboptimal image quality had a higher body mass index. No difference was seen in the presence of systolic dysfunction, coronary artery disease, cardiomyopathy or chronic obstructive lung disease. The LVEFs assessed at the core laboratory ranged from 15 to $75 \%$, the ones assessed at the recruiting centers from 8 to $77 \% .171(41 \%)$ of the patients had a LVEF that was assessed as $\leq 35 \%$ at the core lab. Two hundred nine patients from the original TIME-CHF study population were not included in the present study, either because of the unavailability of digital image sets from two recruiting centers, or because of incomplete imaging datasets. Regarding the basic and clinical parameters, these 209 patients differed from the included patients in that they were younger ( $75.8 \pm 7.6$ vs. $77.5 \pm 7.5$ years, $p=0.007)$, and in that they had a lower proportion of coronary artery disease $(56.9$ vs $68.0 \%, p=0.008)$, but there were no differences regarding BMI, gender, systolic dysfunction, cardiomyopathy, COPD or LVEF as reported by the recruiting center.

\section{Variability in determination of LVEF}

For the whole patient population, there was a highly significant correlation between the two measurements of LVEF with an $\mathrm{R}^{2}$ of 0.62 . However, Bland-Altman analysis showed a wide $95 \%$ confidence interval of the differences ranging from -17.4 to $+17.8 \%$ despite a small overall bias of $0.2 \%$ (Fig. 1), and the variability between the recruiting center and the core lab was $14.1 \pm 10.9 \%$. When the whole patient population was separated into patients with optimal image quality and patients with suboptimal image quality, the correlation for the two measurements remained highly significant for both subpopulations with an $\mathrm{R}^{2}$ of 0.65 in the subpopulation with optimal image quality, and an $\mathrm{R}^{2}$ of 0.59 in the subpopulation with suboptimal image quality. Bland-Altman analysis again showed small biases for both subpopulations $(1.0 \%$ in the subpopulation with good image quality, and $1.3 \%$ in the subpopulation with suboptimal image quality). However, the $95 \%$ confidence intervals were large for both subgroups, though somewhat smaller in the subpopulation with optimal image quality ( $95 \% \mathrm{CI}-16.3$ to $+18.4 \%$ in the subpopulation with optimal image quality, $95 \%$ CI -16.5 to $+19.1 \%$ in the subpopulation with suboptimal image quality) (Fig. 2). Variability between LVEF assessment in the recruiting centers and measurements at the core lab was $14.5 \pm 10.3 \%$ for the subpopulation with optimal image quality, and $13.8 \pm 10.3 \%$ for the subgroup with suboptimal image quality ( $p=0.23$ for the difference between the 2 subgroups).

Overall, the median of the difference between the two LVEF measurements was $5.2 \%$ (IQR 2.5-9.6). Only a

Table 1 Clinical characteristics in the overall study patients and in patients with suboptimal versus good image quality

\begin{tabular}{|c|c|c|c|c|}
\hline & \multirow{2}{*}{$\begin{array}{l}\text { Overall } \\
\mathrm{n}=413\end{array}$} & \multicolumn{2}{|l|}{ Echo quality } & \multirow[t]{2}{*}{$p$} \\
\hline & & $\begin{array}{l}\text { Optimal } \\
\mathrm{n}=191\end{array}$ & $\begin{array}{l}\text { Suboptimal } \\
\mathrm{n}=222\end{array}$ & \\
\hline Age (years \pm SD) & $77.5 \pm 7.5$ & $78.0 \pm 7.3$ & $77.1 \pm 7.7$ & 0.22 \\
\hline $\mathrm{BMI}\left(\mathrm{kg} / \mathrm{m}^{2} \pm \mathrm{SD}\right)$ & $25.4 \pm 4.4$ & $24.5 \pm 4.3$ & $26.5 \pm 4.4$ & $\leq 0.0001$ \\
\hline Male (\%) & $240(58.0)$ & $111(58.1)$ & $129(58.1)$ & 1.0 \\
\hline Systolic dysfunction $(\%)^{\mathrm{a}}$ & $329(79.7)$ & $147(77.0)$ & $182(82)$ & 0.22 \\
\hline CAD & $281(68.0)$ & $126(66.0)$ & $1455(69.8)$ & 0.46 \\
\hline RWMD & $163(39.5)$ & $77(40.3)$ & $85(38.3)$ & 0.78 \\
\hline Cardiomyopathy & $61(14.8)$ & $28(14.7)$ & $33(19.9)$ & 0.87 \\
\hline COPD & $83(20.1)$ & 37 (19.4) & $46(20.7)$ & 0.80 \\
\hline
\end{tabular}

$B M I$ Body mass index, $C A D$ coronary artery disease, $R W M D$ regional wall motion defect, $C O P D$ chronic obstructive pulmonary disease

a Systolic dysfunction defined as LVEF $\leq 45 \%$ 
A

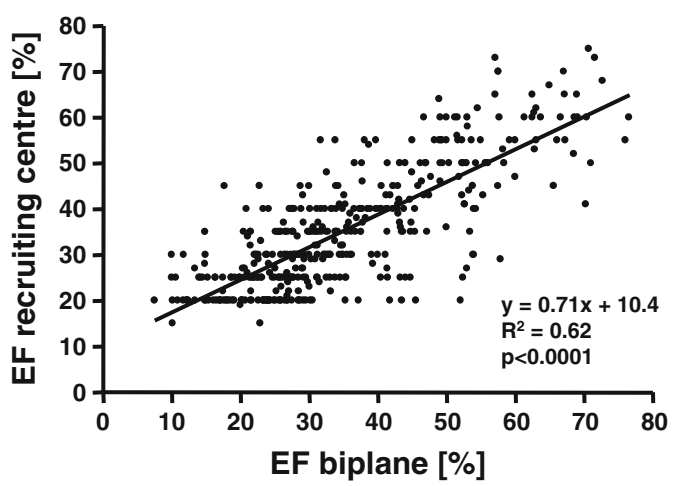

B

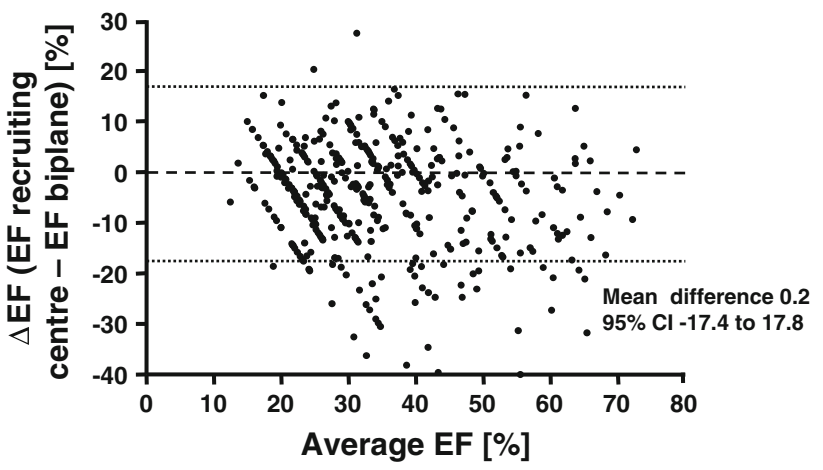

Fig. 1 a Correlation between LVEF measured at the recruiting center and biplane LVEF measured at the core laboratory by linear regression analysis (solid line) for the whole patient group. b Bland-Altmann plot of the same data, dashed lines specify mean difference of the measurements, dotted lines the corresponding $95 \%$ confidence interval (CI) limited number of significant predictors for this difference could be identified in univariate analysis. Thus, it was smaller in male (median [IQR] $4.4 \%$ [2.4-8.6\%]) compared to female (6.6 [2.6-11.1], $p=0.004)$. Furthermore, a larger difference was correlated with higher heart rate $(\mathrm{r}=$ $0.12, p=0.01)$, shorter QRS duration $(\mathrm{r}=-0.11, p=$ 0.03 ), and smaller LV ventricles (end-diastolic volume of LV [LVEDV] $\mathrm{r}=-0.17, p=0.001)$. Other predictors were not significantly correlated with the difference between the two measurements, particularly body mass index, presence of COPD, cause of heart failure, and the centre where initial assessment was done. In multivariate analysis, the only independent predictor was the LVEDV.

163 patients $(39 \%)$ in the whole study population had regional wall motion defects. These patients showed a highly significant correlation between the two measurements of LVEF ( $\mathrm{R}^{2}$ of $\left.0.38, p \leq 0.0001\right)$. Again, BlandAltman analysis showed a wide $95 \%$ confidence interval of the differences ranging from -15.5 to $\pm 17.1 \%$ with an overall bias of $0.8 \%$.

Potential influence of the reliability in determination of LVEF on clinical decision-making

Discrete LVEF thresholds are used for important clinical decisions, and therefore we examined what potential influence the intercenter reliability in the determination of LVEF could have on situating an individual patient above or below commonly used threshold values (Fig. 3). For a
Fig. 2 a Correlation between LVEF measured at the recruiting center and biplane LVEF measured at the core laboratory by linear regression analysis (solid line) for the patients with optimal image quality. b Bland-Altmann plot of the same data, dashed lines specify mean difference of the measurements, dotted lines the corresponding $95 \%$ confidence interval (CI). c Correlation between LVEF measured at the recruiting center and biplane LVEF measured at the core laboratory for the patients with suboptimal image quality. d Bland-Altmann plot of the same data
A

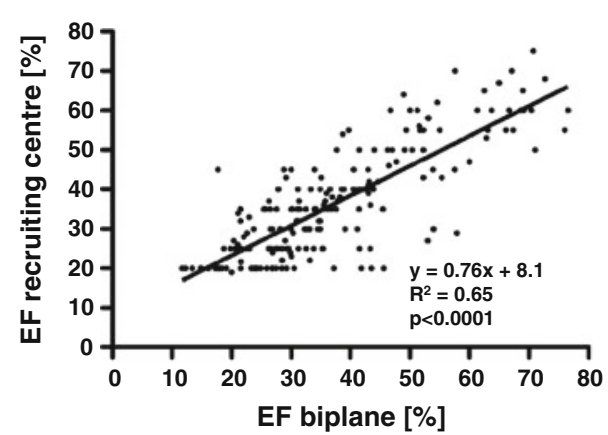

B

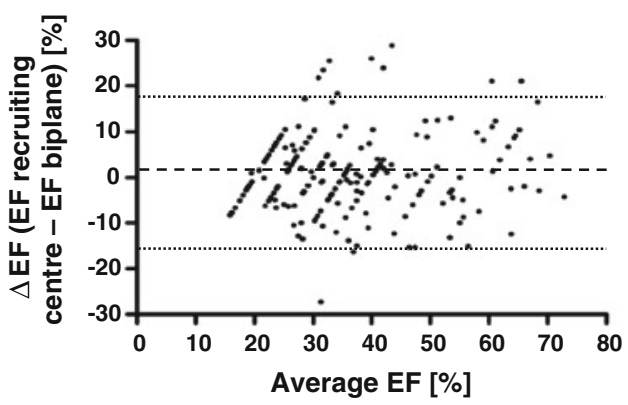

C

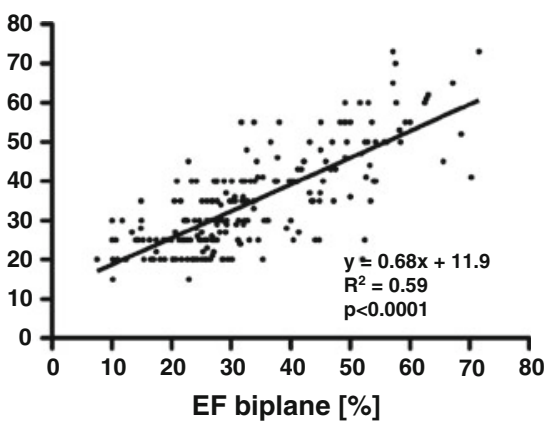

D

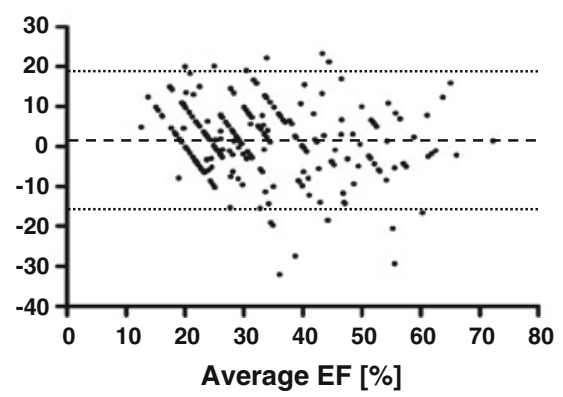


threshold of an LVEF of $30 \%$, in the whole patient population, $21.1 \%$ of all patients changed from either $\leq 30$ to $>30 \%$ or vice versa. For the same threshold, the percentages of re-assignment were $23.0 \%$ for patients with optimal image quality and $19.4 \%$ for patients with suboptimal image quality ( $p$ for the difference in proportions 0.40 ). For a threshold of an LVEF of $\leq 35 \%, 16.9 \%$ were re-assigned in the whole patient population. For patients with optimal image quality the percentage of re-assignment was $16.2 \%$, for patients with suboptimal image quality $17.6 \%$ ( $p$ for the difference in proportions 0.79). For a threshold of an LVEF of $\leq 40 \%, 13.6 \%$ were re-assigned in the whole patient population. For patients with optimal image quality the percentage of re-assignment was $13.1 \%$, for patients with suboptimal image quality $14.1 \%$ ( $p$ for the difference in proportions 0.39 ). For the threshold of a normal LVEF of $\geq 55 \%, 6.3 \%$ were re-assigned in the whole patient population, $5.9 \%$ in the subpopulation with optimal image quality, and $6.8 \%$ in the subpopulation with suboptimal image quality ( $p$ for the difference in proportions 0.69).

\section{Discussion}

Our study shows a high variability in the evaluation of LVEF in a large study of patients with heart failure with both reduced ejection fraction and normal ejection fraction. This high variability resulted despite the fact that the same image material was used for analysis at the recruiting center and at the echocardiography core laboratory. Using commonly accepted cut-offs for the implantation of ICD or CRT, 15-20\% of all patients were re-classified as having a LVEF above or below the cut-offs when the images were re-assessed. Neither the variability in LVEF assessment between hospital centers nor the percentages of patients reclassified depended significantly on the quality of the acquired ultrasound images. The only independent predictor of a high variability in LVEF measurements was a smaller left ventricle.
Interobserver reliability

The numeric assessment of LVEF is the single most important measurement in cardiology with a profound influence on diagnosis and management of patients. Hence, it is of utmost importance that this measurement is reliable and reproducible. While other imaging modalities in use in current cardiology practice (angiography, SPECT, MRI, CT, RNA) can provide measurements of LVEF, two-dimensional echocardiography is by far the most commonly employed method. Two-dimensional echocardiography for the measurement of LVEF relies on either a visual assessment of ventricular function, or on tracings of the endocardial borders and calculation of LVEF using geometric models (Simpson's biplane analysis, area length method). In comparison to other imaging modalities, two-dimensional echocardiography has the disadvantages of (1) dependence on unequivocal endocardial border delineation, which is not uniformly achieved in all echocardiographic images, and (2) reliance on geometric assumptions in the case of LVEF measurement by Simpson's biplane method or area length method. The accuracy of these echocardiographic methods for the assessment of LVEF in comparison with SPECT, MRI and CT has been studied extensively [16-18] and overall correlations have been shown to be good. Thus, 2-dimensional echocardiography is a valuable tool for assessing treatment effects in therapeutic trials. However, there has been concern regarding interstudy, interobserver and intraobserver variability of 2-dimensional echocardiographic measurements of LVEF, especially with regards to the serial assessment of changes in ejection fraction, and inclusion or exclusion of subjects into studies where LVEF thresholds are used as inclusion criteria. By the design of the present study, the LVEF assessment at the recruiting hospital was done either by visual assessment, Simpson's biplane or area length assessment, and thus the $95 \%$ confidence interval of the differences do not represent a strict measurement of interobserver variability. Nevertheless, the variabilities in our study correspond to previously published values of interobserver variability [19,20], while others have published lower values [21-23]. An important difference
Fig. 3 Correlation of LVEF measured at the recruiting center and biplane LVEF measured at the core laboratory for a the whole patient group, b patients with optimal image quality, and $\mathbf{c}$ patients with suboptimal image quality. Those datapoints for which a reclassification from $\leq 30$ to $>30 \%$ occurred are shaded in grey

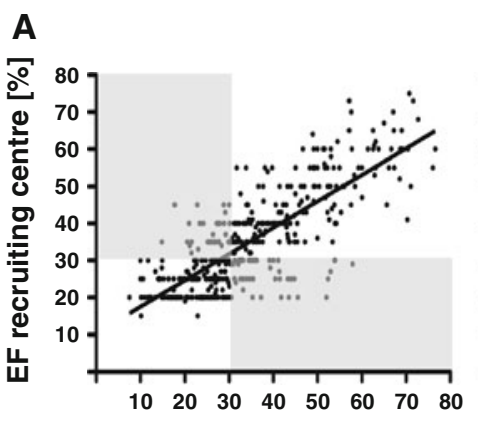

B

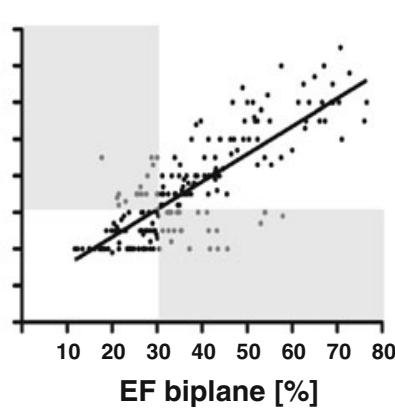

C

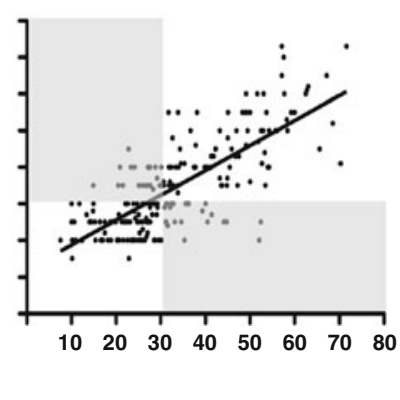


between the cited studies and our data is that we investigated the variability between hospital centers in the assessment of LVEF in a large, real world clinical heart failure trial, while the cited studies were conducted specifically to assess the accuracy and interobserver variability of different imaging methods for the assessment of LVEF in a lower number of subjects. Thus, our study can be assumed to more reliably indicate the variability of LVEF measurements as performed in routine cardiology practice. The variability in the assessment of LVEF in our study was largely independent of the quality of the echocardiographic images. Others have described a better agreement for echocardiography with other imaging techniques in those patients with better image quality [15]. In our study, $53.8 \%$ of patients were deemed to have a suboptimal image quality, which seems to be a relatively high number. The large percentage of patients with suboptimal image quality may be due to the fact that a real world population was examined. More importantly, the assessment of image quality was performed at the core lab to assess its effect on the reliability in measurement of LVEF and reclassification above or below a threshold. To our own surprise, image quality did not have a significant impact on variability, and thus image quality did not impact the main study results. However, our dataset is likely to more closely reflect a real-world situation than data from dedicated imaging studies. Importantly, only a very limited number of factors potentially influencing the variability could be indentified in this study. Of these variables, only the enddiastolic volume of the LV was an independent, albeit very limited predictor.

\section{Reclassification}

A large proportion of patients were reclassified below or above clinically relevant thresholds when the echocardiographic images were reassessed. These proportions were similar for cut-offs of 30, 35, and $40 \%$, all of which are important for clinical decision-making. The proportion of reclassification was lower regarding the cut-off of a normal LVEF, but this seems to be due to the smaller number of patients included in this study with normal LVEF. Previous information on reclassification is very limited. Thus, Chuang et al. compared 2 dimensional echocardiography with CMR with regards to classification of LVEF as normal ( $\geq 55 \%$ ), depressed (LVEF $>35$ to $55 \%$ ) or severely depressed $(\leq 35 \%)$ in a total of 35 patients, and found that up to $44 \%$ of patients were classified differently by echocardiography. Similarly, Ray et al. [24] compared 2D echocardiography to radionuclide ventriculography in 70 patients, and found that $40 \%$ of patients would have been classified differently depending on the imaging test used. Given the far-reaching consequences of placing individuals above or below a certain LVEF threshold, especially with regards to device implantation, the high rate of reclassification in our study is worrying, as it may lead to higher numbers needed to treat both in clinical trials, but also in real world patients. Contrast-enhanced echocardiography and three-dimensional echocardiography have been shown to improve both the accuracy of LVEF determinations when compared to reference methods, as well as to reduce interobserver variability [20, 25]. However, the benefit of these new techniques with regards to misclassification of patients above or below LVEF cutoffs remains to be determined. In addition, our data indicate that studies, especially single center studies, that are specifically designed to test the accuracy and reproducibility of imaging methods, tend to underestimate the measurement variability observed in clinical practice.

\section{Study limitations}

LVEF assessment at the recruiting hospital centers was done according to the preferences of the investigators with either visual assessment or biplane Simpson's method, whereas in the echocardiographic core laboratory all LVEFs were measured using biplane Simpson's method. Thus, we do not report a true interobserver variability. This may have increased the variability we report in this study. Also, the fact that the readers at the core lab, but not the cardiologist at the recruiting center were blinded for the clinical characteristics of the patient might have further increased variability. However, differences in the methodology used for LVEF assessment are the reality in clinical practice, and thus our data closely reflect the true variability of LVEF measurements with two-dimensional echocardiography in daily clinical practice.

\section{Conclusions}

Conventional 2D echocardiographic assessment of LVEF carries with it a considerable variability. This variability is not dependent on overall image quality and identification of factors potentially influencing this variability is very limited. A significant proportion of patients (i.e. 15-20\%) would have been re-assigned to a different LVEF category upon reassessment of the echocardiographic images. Thus, the reported variability in LVEF assessment appears to have an important potential impact on clinical decisionmaking, especially on the indication for the implantation of an ICD or CRT device. Whether the standard use of biplane Simpson's method for LVEF calculations or other imaging modalities would reduce variability of LVEF assessment and its impact on clinical decision-making, and, importantly, would also be applicable in clinical practice remains to be determined. 
Acknowledgments Dr. Kaufmann is supported by a SCORE grant (SNF 32323B_123919/1) from the Swiss National Science Foundation. TIME-CHF was supported by the Horten Research Foundation (Lugano, Switzerland; $>55 \%$ of the study's budget), as well as by smaller unrestricted grants from AstraZeneca Pharma, Novartis Pharma, Menarini Pharma, Pfizer Pharma, Servier, Roche Diagnostics, Roche Pharma, and Merck Pharma.

Conflict of interest None declared.

\section{References}

1. Moss AJ, Zareba W, Hall WJ, Klein H, Wilber DJ, Cannom DS, Daubert JP, Higgins SL, Brown MW, Andrews ML (2002) Prophylactic implantation of a defibrillator in patients with myocardial infarction and reduced ejection fraction. $\mathrm{N}$ Engl $\mathrm{J}$ Med 346:877-883

2. Bardy GH, Lee KL, Mark DB, Poole JE, Packer DL, Boineau R, Domanski M, Troutman C, Anderson J, Johnson G, McNulty SE, Clapp-Channing N, Davidson-Ray LD, Fraulo ES, Fishbein DP, Luceri RM, Ip JH (2005) Amiodarone or an implantable cardioverter-defibrillator for congestive heart failure. N Engl J Med 352:225-237

3. Bristow MR, Saxon LA, Boehmer J, Krueger S, Kass DA, De Marco T, Carson P, DiCarlo L, DeMets D, White BG, DeVries DW, Feldman AM (2004) Cardiac-resynchronization therapy with or without an implantable defibrillator in advanced chronic heart failure. N Engl J Med 350:2140-2150

4. Hunt SA, Abraham WT, Chin MH, Feldman AM, Francis GS, Ganiats TG, Jessup M, Konstam MA, Mancini DM, Michl K, Oates JA, Rahko PS, Silver MA, Stevenson LW, Yancy CW (2009) Focused update incorporated into the acc/aha 2005 guidelines for the diagnosis and management of heart failure in adults: a report of the American college of cardiology foundation/ american heart association task force on practice guidelines: developed in collaboration with the international society for heart and lung transplantation. Circulation 119:e391-e479

5. Dickstein K, Cohen-Solal A, Filippatos G, McMurray JJ, Ponikowski P, Poole-Wilson PA, Stromberg A, van Veldhuisen DJ, Atar D, Hoes AW, Keren A, Mebazaa A, Nieminen M, Priori SG, Swedberg K (2008) Esc guidelines for the diagnosis and treatment of acute and chronic heart failure 2008: the task force for the diagnosis and treatment of acute and chronic heart failure 2008 of the European society of cardiology. Developed in collaboration with the heart failure association of the esc (hfa) and endorsed by the European society of intensive care medicine (esicm). Eur Heart J 29:2388-2442

6. White HD, Norris RM, Brown MA, Brandt PW, Whitlock RM, Wild CJ (1987) Left ventricular end-systolic volume as the major determinant of survival after recovery from myocardial infarction. Circulation 76:44-51

7. Wong M, Johnson G, Shabetai R, Hughes V, Bhat G, Lopez B, Cohn JN (1993) Echocardiographic variables as prognostic indicators and therapeutic monitors in chronic congestive heart failure. Veterans affairs cooperative studies v-heft i and ii. V-heft va cooperative studies group. Circulation 87:VI65-VI70

8. Randomised, placebo-controlled trial of carvedilol in patients with congestive heart failure due to ischaemic heart disease. Australia/new zealand heart failure research collaborative group. Lancet (1997) 349:375-380

9. Effect of enalapril on survival in patients with reduced left ventricular ejection fractions and congestive heart failure. The solvd investigators. N Engl J Med (1991) 325:293-302
10. Effects of metoprolol $\mathrm{cr}$ in patients with ischemic and dilated cardiomyopathy : the randomized evaluation of strategies for left ventricular dysfunction pilot study. Circulation (2000) 101: 378-384

11. Lang RM, Badano LP, Tsang W, Adams DH, Agricola E, Buck T, Faletra FF, Franke A, Hung J, de Isla LP, Kamp O, Kasprzak JD, Lancellotti P, Marwick TH, McCulloch ML, Monaghan MJ, Nihoyannopoulos P, Pandian NG, Pellikka PA, Pepi M, Roberson DA, Shernan SK, Shirali GS, Sugeng L, Ten Cate FJ, Vannan MA, Zamorano JL, Zoghbi WA (2012) Eae/ase recommendations for image acquisition and display using three-dimensional echocardiography. J Am Soc Echocardiogr 25:3-46

12. Pfisterer M, Buser P, Rickli H, Gutmann M, Erne P, Rickenbacher P, Vuillomenet A, Jeker U, Dubach P, Beer H, Yoon SI, Suter T, Osterhues HH, Schieber MM, Hilti P, Schindler R, Brunner-La Rocca HP (2009) Bnp-guided vs symptom-guided heart failure therapy: the trial of intensified vs standard medical therapy in elderly patients with congestive heart failure (time-chf) randomized trial. JAMA 301:383-392

13. Brunner-La Rocca HP, Buser PT, Schindler R, Bernheim A, Rickenbacher P, Pfisterer M (2006) Management of elderly patients with congestive heart failure-design of the trial of intensified versus standard medical therapy in elderly patients with congestive heart failure (time-chf). Am Heart J 151:949-955

14. Lang RM, Bierig M, Devereux RB, Flachskampf FA, Foster E, Pellikka PA, Picard MH, Roman MJ, Seward J, Shanewise JS, Solomon SD, Spencer KT, Sutton MS, Stewart WJ (2005) Recommendations for chamber quantification: a report from the American society of echocardiography's guidelines and standards committee and the chamber quantification writing group, developed in conjunction with the European association of echocardiography, a branch of the european society of cardiology. J Am Soc Echocardiogr 18:1440-1463

15. Tighe DA, Rosetti M, Vinch CS, Chandok D, Muldoon D, Wiggin B, Dahlberg ST, Aurigemma GP (2007) Influence of image quality on the accuracy of real time three-dimensional echocardiography to measure left ventricular volumes in unselected patients: a comparison with gated-spect imaging. Echocardiography 24:1073-1080

16. Erbel R, Schweizer P, Lambertz H, Henn G, Meyer J, Krebs W, Effert S (1983) Echoventriculography—a simultaneous analysis of two-dimensional echocardiography and cineventriculography. Circulation 67:205-215

17. Schalla S, Nagel E, Lehmkuhl H, Klein C, Bornstedt A, Schnackenburg B, Schneider U, Fleck E (2001) Comparison of magnetic resonance real-time imaging of left ventricular function with conventional magnetic resonance imaging and echocardiography. Am J Cardiol 87:95-99

18. Bellenger NG, Burgess MI, Ray SG, Lahiri A, Coats AJ, Cleland JG, Pennell DJ (2000) Comparison of left ventricular ejection fraction and volumes in heart failure by echocardiography, radionuclide ventriculography and cardiovascular magnetic resonance; are they interchangeable? Eur Heart J 21:1387-1396

19. Chuang ML, Hibberd MG, Salton CJ, Beaudin RA, Riley MF, Parker RA, Douglas PS, Manning WJ (2000) Importance of imaging method over imaging modality in noninvasive determination of left ventricular volumes and ejection fraction: assessment by two- and three-dimensional echocardiography and magnetic resonance imaging. J Am Coll Cardiol 35:477-484

20. Hoffmann R, von Bardeleben S, ten Cate F, Borges AC, Kasprzak J, Firschke C, Lafitte S, Al-Saadi N, Kuntz-Hehner S, Engelhardt M, Becher H, Vanoverschelde JL (2005) Assessment of systolic left ventricular function: a multi-centre comparison of cineventriculography, cardiac magnetic resonance imaging, unenhanced and contrast-enhanced echocardiography. Eur Heart J 26: $607-616$ 
21. Whalley GA, Gamble GD, Walsh HJ, Wright SP, Agewall S, Sharpe N, Doughty RN (2004) Effect of tissue harmonic imaging and contrast upon between observer and test-retest reproducibility of left ventricular ejection fraction measurement in patients with heart failure. Eur J Heart Fail 6:85-93

22. Shahgaldi K, Gudmundsson P, Manouras A, Brodin LA, Winter $R$ (2009) Visually estimated ejection fraction by two dimensional and triplane echocardiography is closely correlated with quantitative ejection fraction by real-time three dimensional echocardiography. Cardiovasc Ultrasound 7:41

23. Cannesson M, Tanabe M, Suffoletto MS, McNamara DM, Madan S, Lacomis JM, Gorcsan J III (2007) A novel two-dimensional echocardiographic image analysis system using artificial intelligence-learned pattern recognition for rapid automated ejection fraction. J Am Coll Cardiol 49:217-226

24. Ray SG, Metcalfe MJ, Oldroyd KG, Pye M, Martin W, Christie J, Dargie HJ, Cobbe SM (1995) Do radionuclide and echocardiographic techniques give a universal cut off value for left ventricular ejection fraction that can be used to select patients for treatment with ace inhibitors after myocardial infarction? $\mathrm{Br}$ Heart J 73:466-469

25. Jenkins C, Bricknell K, Hanekom L, Marwick TH (2004) Reproducibility and accuracy of echocardiographic measurements of left ventricular parameters using real-time threedimensional echocardiography. J Am Coll Cardiol 44:878-886 